\title{
Can gauge theories of flavour be accessible at the LHC?
}

\section{Benedetta Belfatto*}

Gran Sasso Science Institute, Viale Francesco Crispi 7, 67100 LAquila (AQ), Italy INFN, Laboratori Nazionali del Gran Sasso, Assergi, 67100 L'Aquila (AQ), Italy

E-mail: benedetta.belfattodgssi.it

\begin{abstract}
Horizontal gauge symmetries between fermion families and their spontaneous breaking can be at the origin of the inter-family mass hierarchies. The corresponding gauge bosons must have flavor changing couplings to fermions and generically the very stringent limits on their masses arise from flavour changing phenomena and CP-violation. However, in the special cases of chiral horizontal symmetries separately acting on left and right species, manifesting suppression effects due to custodial symmetry, the flavor-changing bosons acting between first two families are allowed to be as light as few $\mathrm{TeV}$, without contradiction with the existing experimental limits and thus they can be detectable as new resonances at the LHC.
\end{abstract}

The European Physical Society Conference on High Energy Physics 5-12 July

Venice, Italy

${ }^{*}$ Speaker. 
The replication of fermion families is one of the main puzzles of particle physics. All known particle species are described by Standard Model (SM) $S U(3) \times S U(2) \times U(1)$, and its electroweak (EW) part is chiral with respect to fermion multiplets. In other words, three families of the left -handed (L) quark and lepton fields $q_{L}^{i}=\left(u^{i}, d^{i}\right)_{L}, l_{L}^{i}=\left(v^{i}, e^{i}\right)_{L}$ form weak doublets while the righthanded components $u_{R}^{i}, d_{R}^{i}, e_{R}^{i}$ are singlets, where $i=1,2,3$ is the family index. The chiral fermion content of SM has remarkable feature that fermion masses can emerge only after spontaneous breaking of EW gauge part, via the fermion Yukawa couplings $Y_{i j}^{f}$ with the Higgs field $\phi$ which also breaks $S U(2) \times U(1)$ down to $U(1)_{\mathrm{EM}},\left\langle\phi^{0}\right\rangle=v_{\mathrm{EW}}=174 \mathrm{GeV}$. The fermion mass matrices $M^{f}=Y^{f} v_{\mathrm{EW}}, f=u, d, e, v$, can be diagonalized via bi-unitary transformations $V_{f R}^{\dagger} M^{f} V_{f L}=M_{\mathrm{diag}}^{f}$. The known masses of quarks and leptons, $m_{e}, m_{u}, m_{d} \ldots m_{\tau}, m_{b}, m_{t}$, are the eigenvalues of these mass matrices, while the CKM mixing matrices of quarks and neutrinos in charged weak currents are respectively $V_{q L}=V_{u L}^{\dagger} V_{d L}$ and $V_{l L}=V_{v L}^{\dagger} V_{e L}$. Remarkably, no mixing emerges at tree level in the neutral currents, while the Yukawa matrices $Y^{f}$ become diagonal together with mass matrices. In this way, no flavor changing effects emerge at the tree-level in the fermion couplings to $Z$-boson and Higgs boson, remarkable feature known as natural suppression of flavor-changing neutral currents (FCNC) in the SM [1]. However, SM itself tells nothing about the structure of the Yukawa coupling matrices $Y_{i j}^{f}$ which remain arbitrary, and thus SM has no answer on the origin of fermion mass hierarchy and structure of weak mixing angles.

On the other hand, the fermion mass spectrum and weak mixing pattern indicate towards interesting features. Namely, the inter-family hierarchy between the charged lepton and down quark species can be parametrised by a small parameter $\varepsilon \sim 1 / 20$ or so, while the mass spectrum of up quarks shows much stronger hierarchy:

$$
m_{e}: m_{\mu}: m_{\tau} \sim m_{d}: m_{s}: m_{b} \sim \varepsilon^{2}: \varepsilon: 1, \quad m_{u}: m_{c}: m_{t} \sim \varepsilon^{4}: \varepsilon^{2}: 1
$$

On the other hand, one can observe that the CKM angles of quark mixing in $V_{q L}$ are small:

$$
\sin \theta_{12}^{q} \sim \sin \theta_{23}^{q} \sim \varepsilon, \quad \sin \theta_{13}^{q} \sim \varepsilon^{2}
$$

while the neutrino mixing angles are large and presumably there is no significant hierarchy between the neutrino masses.

The key towards understanding the fermion mass and mixing pattern can be in symmetry principles. One can consider e.g. the situation in which different fermion species are separated by different charges of some extra $U(1)$ flavor symmetries [2]. Alternatively, one can consider nonabelian horizontal gauge symmetries $S U(3)$ or $U(3)$ unifying different families [3, 4]. It is suggestive to think that in this case the fermion mass hierarchy and mixing pattern should be related to the structure of vacuum expectation values (VEV) of horizontal "flavon" scalar fields that break this symmetry, coined as hypothesis of horizontal hierarchies (HHH) in Ref. [4]. This hypothesis, in particular, implies that the horizontal family symmetry should have a chiral character, transforming left and right particle species in different representations as the SM itself, and thus fermions should not be allowed to get masses until this gauge symmetry is spontaneously broken. This means that fermion masses should emerge via higher order operators containing flavon scalars, which operators in the context of UV-complete theories can be induced via renormalizable interactions after integrating out some heavy messenger fields, scalars [3] or verctor-like fermions [4]. 
In the context of the SM, the maximal chiral symmetry is $U(3)^{5}$, transforming species $q, u, d, l$ and $e$ as triplets of independent groups $U(3)_{q} \times U(3)_{u} \times U(3)_{d} \times U(3)_{l} \times U(3)_{e}$ :

$$
q_{L i} \sim 3_{q}, \quad u_{R}^{j} \sim \overline{3}_{u}, \quad d_{R}^{\alpha} \sim \overline{3}_{d}, \quad l_{L \beta} \sim 3_{l}, \quad e_{R}^{k} \sim \overline{3}_{e} .
$$

In the context of grand unification $S U(5)$, the choice is restricted to $U(3)^{2}=U(3)_{l} \times U(3)_{e}$, since fermion species are unified in $\overline{5}$-plets $\left(l, d^{c}\right)_{\beta L}$ and 10-plets $\left(q, u^{c}, e^{c}\right)_{i L}$, so that we would have $q_{i}, \bar{u}_{i}, \bar{e}_{i} \sim 3_{e} l_{\alpha}, \bar{d}_{\alpha} \sim 3_{l}$. We consider a situation when only non-abelian parts $S U(3)$ of horizontal $U(3)$ 's are gauged while abelian $U(1)$ factors remain as global symmetries giving rise to Goldstone bosons as axion, majoron and familon with interesting phenomenological implications [5]. In the context of supersymmetry, such $S U$ (3) symmetries can lead to interesting relations between particle and sparticle mass spectra and naturally realize the minimal flavor violation scenarios $[6,7]$.

Let us remark that promoting non-abelian factors $S U(3)_{e}$, etc. as gauge symmetries, one has to take care of anomaly cancellations. One can introduce some ad hoc fermions [3, 4]. Most interesting way is to introduce a hidden particle sector, as a mirror copy of the SM described by the gauge symmetry $S U(3)^{\prime} \times S U(2)^{\prime} \times U(1)^{\prime}$ with particle content identical to ordinary one, modulo the difference that while in the SM ordinary particles are left-handed: $q_{L}, u_{R}, d_{R}$ etc., the mirror sector has identical Lagrangian in the basis of right-handed mirror particles $q_{R}^{\prime}, u_{L}^{\prime}, d_{L}^{\prime}$ etc. In this way, the parity can be understood as a discrete symmetry of exchange between ordinary and mirror species, $q_{L} \leftrightarrow q_{R}^{\prime}$ etc. with respective exchange of ordinary and mirror gauge bosons and Higgses. In this case, independently of whether mirror symmetry is exact or spontaneously broken (so that mirror particles become heavier than ordinary ones [8]), if the gauge symmetries $S U(3)_{e}, S U(3)_{l}$ etc. are acting between two sectors, their gauge anomalies reciprocally cancelled between ordinary and mirror fermions $l_{L \alpha} \sim 3_{l}$ and $l_{R \alpha}^{\prime} \sim 3_{l}, e_{R i} \sim 3_{e}$ and $e_{L i}^{\prime} \sim 3_{e}$, etc. [6]. Thus, horizontal gauge bosons can be messengers between ordinary and mirror sectors and, provided that they are light enough, can give an interesting portal for direct detection of mirror dark matter, which also has many interesting astrophysical and cosmological implications (for review see e.g. [9]).

Spontaneous breaking of family symmetry can be obtained via flavon fields which are the gauge singlets with respect to the SM. For simplicity, for fully breaking $U(3)_{l} \times U(3)_{e}$, one can take three scalar triplets $\xi_{n}^{i} \sim \overline{3}_{e}$ of $S U(3)_{l}$ and three scalar triplets $\eta_{n}^{a} \sim \overline{3}_{l}$ of $S U(3)_{e}, n=1,2,3$. The fermion masses then emerge via gauge invariant higher order effective operators:

$$
\mathscr{L}=\sum_{n}\left[\frac{\xi_{n}^{i} \xi_{n}^{j}}{M^{2}} \bar{\phi} \bar{u}_{j} q_{i}+\frac{\eta_{n}^{\alpha} \xi_{n}^{i}}{M^{2}} \phi \bar{d}_{\alpha} q_{i}+\frac{\xi_{n}^{i} \eta_{n}^{\alpha}}{M^{2}} \phi \bar{e}_{i} l_{\alpha}+\frac{\eta_{n}^{\alpha} \eta_{n}^{\beta}}{\mathscr{M} M^{2}} \bar{\phi} \bar{\phi} l_{\alpha} l_{\beta}\right]+\text { h.c. }
$$

which can be induced via 'universal seesaw' mechanism [4] via integrating out some extra heavy vector-like fermions with quantum numbers of quarks and leptons with masses $\sim M$ and, for the case of neutrino, with Majorana mass $\mathscr{M} \gg M$.

Yukawa couplings of the SM will be obtained after SSB of the family symmetries. Let us assume that three flavon triplets $\xi_{n}$ of $S U(3)_{e}$ have disoriented VEVs

$$
\left\langle\xi_{3}\right\rangle=v_{3}\left(\begin{array}{l}
0 \\
0 \\
1
\end{array}\right), \quad\left\langle\xi_{2}\right\rangle=v_{2}\left(\begin{array}{l}
0 \\
1 \\
x
\end{array}\right), \quad\left\langle\xi_{1}\right\rangle=v_{1}\left(\begin{array}{l}
1 \\
y \\
z
\end{array}\right)
$$


having a hierarchy between the VEVs $v_{1}: v_{2}: v_{3} \sim \varepsilon^{2}: \varepsilon: 1$ with $\varepsilon \sim 1 / 20$ or so, and $x, y, z$ are order 1. As for flavons $\eta_{n}$ breaking $S U(3)_{l}$, they can have also disoriented VEVs of similar structure but with values of the same order, $u_{1,2,3} \sim v_{3}$. In this case, from the couplings (4) we would obtain the fermion mass matrices which would explain the fermion mass and mixing pattern (1) and (2).

Let us question now, how small the masses of horizontal gauge bosons can be without contradicting to experimental limits. ${ }^{1}$ Here concentrate on the lepton sector, with horizontal gauge symmetries $S U(3)_{l} \times S U(3)_{e}$, and show that flavor gauge bosons of $S U(3)_{e}$ interacting with righthanded leptons can be as light as few TeV. The reason is that after breaking of $S U(3)_{e}$ by the VEV $v_{3}$, the residual to $S U(2)_{e}$ gauge subgroup exhibits an approximate custodial symmetry which suppresses the FCNC phenomena at the needed level [11].

Exchange of flavor gauge bosons lead to four-fermion (current $\times$ current) interactions:

$$
\mathscr{L}_{e f f}=g_{e}^{2} J_{a}^{(f)}\left(M_{\Theta}^{2}\right)_{a b}^{-1} J_{b}^{(f)} \quad J_{a}^{(e) \mu}=\frac{1}{2}(\bar{e} \bar{\mu} \bar{\tau})_{R} \gamma^{\mu} V_{R}^{(e) \dagger} \lambda_{a} V_{R}^{(e)}\left(\begin{array}{c}
e \\
\mu \\
\tau
\end{array}\right)_{R}
$$

where $\Theta_{a}, a=1,2, . .8$, are the $S U(3)_{e}$ gauge bosons and $M_{\Theta}^{2}$ is their mass matrix induced by the VEVs (5). In order to better understand the protective power of custodial symmetry, it is useful to consider first the limit $v_{3} \rightarrow \infty$ in the breaking of $S U(3)_{e}$. This VEV reduces $S U(3)_{e}$ to $S U(2)_{e}$ subgroup, 5 flavor bosons acting on third family become heavy and decouple, while third family gets a mass. Thus, at low energies there remains only the gauge $S U(2)_{e}$ acting between first two families which will have equal masses $M_{a}^{2}=g v_{2}^{2} / 2, a=1,2,3$ inducing effective operators

$$
\frac{1}{4 v_{2}^{2}}\left(\bar{f}_{R} \tau^{a} \gamma^{\mu} f_{R}\right)\left(\bar{f}_{R} \tau^{a} \gamma_{\mu} f_{R}\right)=\frac{1}{4 v_{2}^{2}}\left(\bar{e}_{1} \gamma_{\mu} e_{1}+\bar{e}_{2} \gamma_{\mu} e_{2}\right)^{2}
$$

where $f_{R}=\left(e_{1}, e_{2}\right)_{R}$ and $\tau^{a}$ are the Pauli matrices, mediated by gauge bosons $\Theta_{1,2,3}$, or in equivalent form, $\theta_{1}^{2}=\frac{1}{\sqrt{2}}\left(\theta_{1}-i \theta_{2}\right), \theta_{2}^{1}=\frac{1}{\sqrt{2}}\left(\theta_{1}+i \theta_{2}\right)$ and $\theta_{3}$. Obviously, no FCNC emerge in this limit. This suppression effect of $S U(2)$ symmetry is related to custodial symmetry.

Considering $\mathrm{SU}(3)_{e}$ gauge bosons involving third family, flavour changing neutral currents can emerge at tree level by two different reasons: 1 ) the heavy gauge bosons can be mixed with the lighter ones $\Theta_{1,2,3}$ in the mass matrix 2) the lepton fields $e_{1}$ and $e_{2}$ in the $S U(2)_{e}$ doublet do not coincide with the mass eigenstates $e$ and $\mu$, and the mixing with $\tau$ lepton will induce the FCNC:

$$
\left(\begin{array}{c}
e^{1} \\
e^{2} \\
e^{3}
\end{array}\right)_{R}=V_{R}^{(e)}\left(\begin{array}{c}
e \\
\mu \\
\tau
\end{array}\right)_{R}=\left(\begin{array}{lll}
V_{1 e} & V_{1 \mu} & V_{1 \tau} \\
V_{2 e} & V_{2 \mu} & V_{2 \tau} \\
V_{3 e} & V_{3 \mu} & V_{3 \tau}
\end{array}\right)\left(\begin{array}{c}
e \\
\mu \\
\tau
\end{array}\right)_{R}
$$

where $\left|V_{1 \mu}\right| \sim\left|V_{2 e}\right| \sim \varepsilon$ and $\left|V_{1 \tau}\right| \sim\left|V_{3 e}\right| \sim \varepsilon^{2}$. As we show now, both contributions are suppressed in relevant processes by respective powers of $\varepsilon$ parameter and thus the masses of $S U(2)_{e}$ gauge bosons are allowed to be of the order of $\mathrm{TeV}$.

\footnotetext{
${ }^{1}$ In the context of the models $[3,4,5]$ this scale was considered to be many orders of magnitude larger than the electroweak scale, with $v_{2}>10^{6} \mathrm{GeV}$ or so, for avoiding excessive flavor changing in neutral currents. For exception, see however Ref. [10].
} 
The contributions of the new operators to flavour changing processes must be computed and compared with the Standard Model predictions and experimental results. In particular the following flavour changing operators involving muons will be considered.

$$
-2 \frac{G_{\mu e e e}}{\sqrt{2}} \overline{e_{R}} \gamma^{\mu} \mu_{R} \overline{e_{R}} \gamma^{\mu} e_{R} \quad \mu \rightarrow e e e \quad-2 \frac{G_{\mu e \mu e}}{\sqrt{2}} \overline{e_{R}} \gamma^{\mu} \mu_{R} \overline{e_{R}} \gamma^{\mu} \mu_{R} \quad \mu \bar{e} \rightarrow e \bar{\mu}
$$

where

$$
-2 \frac{G_{\mu e e e}}{\sqrt{2}}=\frac{1}{2 v_{2}^{2}} V_{3 e}^{*} V_{3 \mu}
$$

By using the experimental limits from [12], [13]

$$
\left|G_{\mu e \mu e}\right| / 2 G_{F}<3 \cdot 10^{-3}, \quad \Gamma(\mu \rightarrow e e e) / \Gamma_{\text {total }}<10^{-12} \rightarrow\left|G_{\mu e e e}\right| / 2 G_{F}<10^{-6}
$$

and it is obtained that

$$
v_{2}>10^{3} \varepsilon^{3 / 2} v_{E W} \sim \mathrm{TeV}
$$

Analogous operators arise for the $\tau$ lepton family violating decay modes. By using the experimental limits from [14] it is obtained that $v_{2}>\varepsilon^{1 / 2} 5 \mathrm{TeV}$.

Compositeness limits on the operators $-\frac{2 \pi}{\Lambda^{2}} \bar{e}_{R} \gamma_{\mu} e_{R} \bar{e}_{R} \gamma^{\mu} e_{R}$, etc. must also be respected ([15], [16]):

$$
v_{2}=\frac{\Lambda_{R R}^{-}(e e e e)}{2 \sqrt{2 \pi}}>2 \mathrm{TeV} \quad v_{2}=\frac{\Lambda_{R R}^{-}(e e \mu \mu)}{2 \sqrt{\pi}}>2.6 \mathrm{TeV}
$$

If $v_{3}$ is big but finite, then the mixing of gauge bosons must be considered. However the matrix elements $M_{a b}^{-2} \propto 1 / v_{3}^{2}$ for $a, b \neq 1,2$, so this contribution is suppressed with respect to the contribution belonging to the mixing of fermions.

In the presence of mirror sector, the effective flavor changing operator which goes unsuppressed, is $\frac{1}{v_{2}^{2}}\left(\bar{e}_{R} \gamma^{\mu} \mu_{R}\right)\left(\bar{\mu}_{L}^{\prime} \gamma^{\mu} e_{L}^{\prime}\right)$ which would lead to muonium oscillation phenomenon into mirror muonium, its mass degenerate mirror partner: $M\left(\mu^{+} e^{-}\right) \rightarrow M^{\prime}\left(\bar{\mu}^{\prime} e^{\prime}\right)$. While the present limit on the muonium disappearance $\operatorname{Br}(M \rightarrow$ invisible $)<5.7 \times 10^{-6}$ [21] is respected for $v_{2} \sim 400 \mathrm{GeV}$ or so, the future experiments on this phenomenon reaching the sensitivity $\sim 10^{-12}$ [21] could reveal this interesting portal to mirror sector.

The same discussion can be applied to quark sector, and it can be shown that also the $S U(2)_{q}$ gauge bosons acting between first and second families can be as light as few TeV. However, in the $S U(5)$ motivated context, when $S U(3)_{q}$ acts also on $\bar{u}$ and $\bar{e}$ in the same multiplet as $q$, so the limits on semileptonic flavor violating decays of kaons and $K^{0}$ and B-mesons oscillations must be checked and generically more stringent limits on $v_{2}$ will be obtained.

Concluding, we have shown that the scale $v_{2}$ originating the mass of the horizontal gauge bosons mediating flavour changing transitions between families can be as light as few $\mathrm{TeV}$, without contradicting the experimental limits of the flavour changing. Since $M_{\Theta}^{2}=\frac{v_{2}^{2} g^{2}}{2}$ and $g \lesssim 1$, the mass of the gauge bosons $\Theta_{1,2,3}$ can be lighter than TeV. Apart of inducing interesting FCNC phenomena which might be responsible for some observed anomalies in $B$ decays, these bosons could be also mediators for direct detection of mirror dark matter, and perhaps they can be detected as new resonances at the LHC.

I thank Zurab Berezhiani for collaboration and useful advices. 


\section{References}

[1] S. L. Glashow and S. Weinberg, Phys. Rev. D 15, 1958 (1977); E. A. Paschos, Phys. Rev. D 15, 1966 (1977).

[2] C. D. Froggatt and H. B. Nielsen, Nucl. Phys. B 147, 277 (1979).

[3] Z. G. Berezhiani and J. L. Chkareuli, Sov. J. Nucl. Phys. 37, 618 (1983). JETP Lett. 35, 612 (1982); JETP Lett. 37, 338 (1983).

[4] Z. G. Berezhiani, Phys. Lett. 129B, 99 (1983); Phys. Lett. 150B, 177 (1985).

[5] Z. G. Berezhiani and M. Y. Khlopov, Z. Phys. C 49, 73 (1991); Sov. J. Nucl. Phys. 51, 739 (1990); Sov. J. Nucl. Phys. 51, 935 (1990); Sov. J. Nucl. Phys. 52, 60 (1990); Sov. J. Nucl. Phys. 52, 344 (1990); Z. G. Berezhiani, A. S. Sakharov and M. Y. Khlopov, Sov. J. Nucl. Phys. 55, 1063 (1992).

[6] Z. Berezhiani, Phys. Lett. B 417, 287 (1998).

[7] Z. Berezhiani, Nucl. Phys. Proc. Suppl. 52A, 153 (1997); A. Anselm and Z. Berezhiani, Nucl. Phys. B 484, 97 (1997); Z. Berezhiani and A. Rossi, Nucl. Phys. Proc. Suppl. 101, 410 (2001).

[8] Z. Berezhiani and R. N. Mohapatra, Phys. Rev. D 52, 6607 (1995); Z. Berezhiani, A. D. Dolgov and R. N. Mohapatra, Phys. Lett. B 375, 26 (1996); Z. Berezhiani, Acta Phys. Polon. B 27, 1503 (1996).

[9] Z. Berezhiani, Int. J. Mod. Phys. A 19, 3775 (2004), hep-ph/0508233; Eur. Phys. J. ST 163, 271 (2008).

[10] Z. G. Berezhiani and J. L. Chkareuli, Sov. J. Nucl. Phys. 52, 383 (1990).

[11] Z. Berezhiani and G. Dvali, Phys. Lett. B 450, 24 (1999).

[12] L. Willmann et al., Phys. Rev. Lett. 82, 49 (1999).

[13] U. Bellgardt et al. [SINDRUM Collaboration], Nucl. Phys. B 299, 1 (1988).

[14] K. Hayasaka et al., Phys. Lett. B 687, 139 (2010).

[15] D. Bourilkov, Phys. Rev. D 64, 071701 (2001).

[16] S. Schael et al. [ALEPH Collaboration], Eur. Phys. J. C 49, 411 (2007).

[17] S. Aoki et al., Eur. Phys. J. C 74, 2890 (2014).

[18] A. Sher et al., Phys. Rev. D 72, 012005 (2005).

[19] R. Appel et al., Phys. Rev. Lett. 85, 2877 (2000).

[20] N. Carrasco et al. [ETM Collaboration], JHEP 1403, 016 (2014).

[21] S. N. Gninenko, N. V. Krasnikov and V. A. Matveev, Phys. Rev. D 87, 015016 (2013) 